\title{
Variability in Blood Pressure Measurements from Recorded Auscultation Sounds
}

\author{
Alan Murray ${ }^{1}$, Dingchang Zheng ${ }^{2}$, Chengyu Liu ${ }^{3}$, David Graham ${ }^{1}$, Jeff Neasham ${ }^{1}$, \\ Adrian Cossor ${ }^{4}$, Clive Griffiths ${ }^{1}$ \\ ${ }^{1}$ Newcastle University, UK \\ ${ }^{2}$ Anglia Ruskin University, UK \\ ${ }^{3}$ Southeast University, Nanjing, China \\ ${ }^{4} \mathrm{AC}$ Cossor \& Son (Technology) Ltd, UK
}

\begin{abstract}
Blood pressure measurement is clinically important. The manual auscultation method is retained as the current "gold standard". This study was designed to evaluate the variability of repeat measurements by the same operator, and between operators, when evaluating the same data.

Ten young volunteer subjects with no known cardiovascular disease were studied. Korotkoff sounds were recorded from a standard stethoscope head connected to an acoustic microphone, and the audio sounds recorded during blood pressure measurements with cuff deflation, while subjects sat quietly on a chair. All recordings were replayed blindly and independently, to two trained operators. The operators identified systolic and diastolic blood pressure (SBP and DBP) without knowledge of the subject. After all recordings had been analysed once, the analysis was repeated.

$S B P$ ranged from 95 to $112 \mathrm{mmHg}$, and $\mathrm{DBP}$ from 58 to $78 \mathrm{mmHg}$. The difference in repeat listening measurements for each observer was $-0.8 \pm 3.3$ and $-0.5 \pm 2.1 \mathrm{mmHg}$ for $\mathrm{SBP}$, and $-0.4 \pm 2.3$ and $0.4 \pm 1.7 \mathrm{mmHg}$ for $\mathrm{DBP}$. The difference between the two operators for each measurement was $0.7 \pm 1.6$ and $0.9 \pm 3.6 \mathrm{mmHg}$ for $S B P$, and $-1.7 \pm 2.3$ and $-1.0 \pm 2.0 \mathrm{mmHg}$ for $\mathrm{DBP}$. We have shown similar variability between operators as between repeat measurements on identical recordings.
\end{abstract}

\section{Introduction}

The successful measurement of blood pressure with an occlusion cuff was advanced considerably after the publication and description of the classical sounds that are detected over the brachial artery during controlled deflation of the cuff around the upper arm. These sounds were subsequently termed Korotkoff sounds, named after the doctor from St Petersburg who published his description in 1906 [1].

Much clinical training has gone into the manual recognition of these sounds, but with the pervasive use of automated devices it is now felt by some in the clinical community that the ability to measure blood pressure (BP) with a stethoscope and cuff is being lost [2].

Graves and Sheps refer to specific measurement difficulties and emphasise that "Ample evidence has shown that physicians have not been adequately trained to measure $\mathrm{BP}$ and, therefore, rarely measure $\mathrm{BP}$ to the standards asked" [3].

Confidence is needed to show that blood pressure can, with suitable training, be measured with good repeatability and low variability for blood pressure measurements in individual subjects.

\subsection{Reference measurements}

Nevertheless, for the evaluation of automated blood pressure devices, a reference standard is required, and auscultation with a standard stethoscope is still the accepted method, and is referred to as the "gold standard". All published device evaluation standards have used this gold standard, as does the current international standard from the International Organisation for Standardisation (ISO), developed with the assistance of the European Hypertension Society and American Association for Medical Instrumentation.

This international standard requires comparative measurements using the manual blood pressure measurement technique $[4,5]$.

\subsection{Aim of study}

The aim of this study was to evaluate variability in the measurement technique, using carefully recorded Korotkoff sounds so they could be replayed more than once, enabling repeatability of measurements to be evaluated, including for more than one operator.

\section{Recording of auscultation sounds}




\subsection{Subjects}

Ten young volunteer subjects with no known cardiovascular disease were studied. Since they were young, this has a bearing on the range of blood pressure measured.

The study received ethical permission from the Newcastle \& North Tyneside Research Ethics Committee. The investigation conformed with the principles in the Declaration of Helsinki.

\subsection{Recording of Korotkoff sounds}

Korotkoff sounds were recorded (figure 1) from a standard stethoscope head connected to an acoustic microphone, and the audio sounds were recorded to a computer during blood pressure measurements with cuff deflation, while subjects sat quietly on a chair.

The cuff pressure was recorded simultaneously to the computer.

\section{Analysis}

\subsection{Determining blood pressure}

All recordings were replayed blindly and independently, to two trained operators. Both operators used the same computer replay software and the same high quality headphones (Sony MDR-ZX100, $12 \mathrm{~Hz}$ to $22 \mathrm{kHz}$ frequency range).

The operators identified systolic and diastolic blood pressure (SBP and DBP) manually without knowledge of the subject.
Analysis of all recordings was repeated on two completely separate sessions to ensure that there was no memory of the first analysis. All recordings were analysed once, and then the analysis was repeated a second time.

\subsection{Operator training}

The Korotkoff sounds were analysed by two experienced observers. Both observers had successfully completed the British and Irish Hypertension Society auscultatory measurement training package [6]. [https://bihsoc.org/resources/bp-measurement/bpmeasurement-auscultatory-tutorials/]

\section{4. $\quad$ Results}

\subsection{Average blood pressure measurements}

For all individual blood pressure results, including repeat measurements, $\mathrm{SBP}$ ranged from 95 to $112 \mathrm{mmHg}$ and DBP from 58 to $78 \mathrm{mmHg}$.

Figure 2 shown the average values of SBP and DBP for each subject, averaged across both operators and both measurements.

\subsection{Repeat measurements}

The difference in repeat listening measurements for each operator was $-0.8 \pm 3.3$ and $-0.5 \pm 2.1 \mathrm{mmHg}$ for SBP, and $-0.4 \pm 2.3$ and $0.4 \pm 1.7 \mathrm{mmHg}$ for DBP (all mean \pm $\mathrm{SD}$, standard deviation). This is shown in figure 3 .

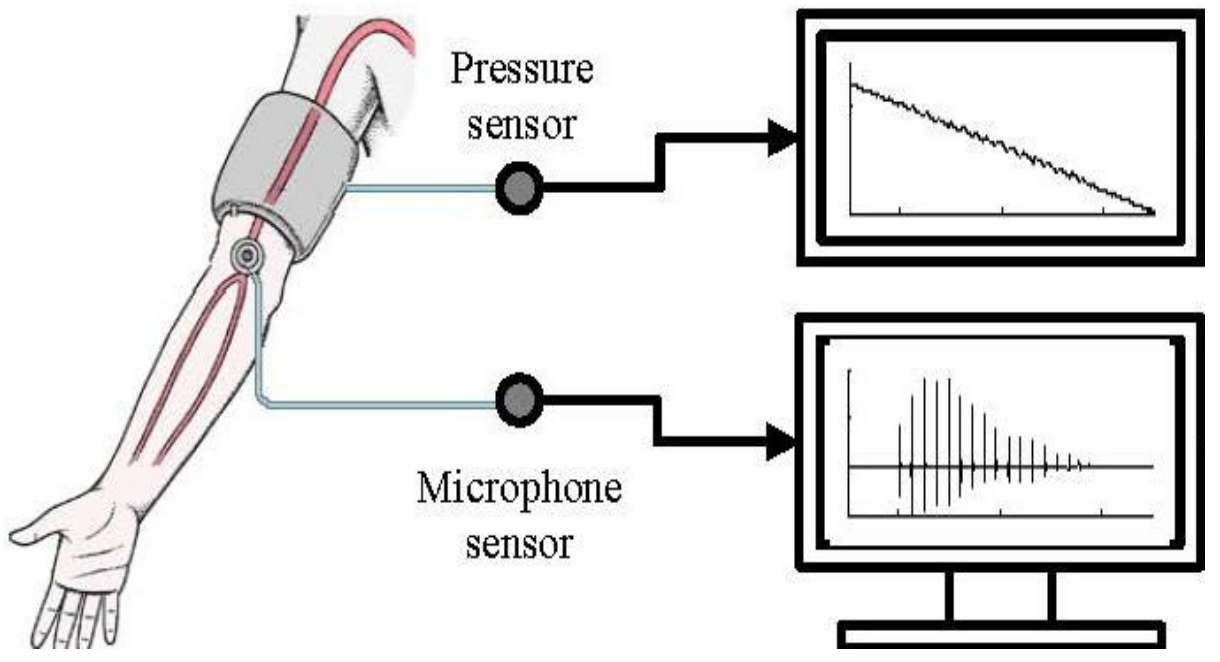

Figure 1. Recording of cuff pressure with a low frequency sensor, and stethoscope acoustic sounds with a microphone. 


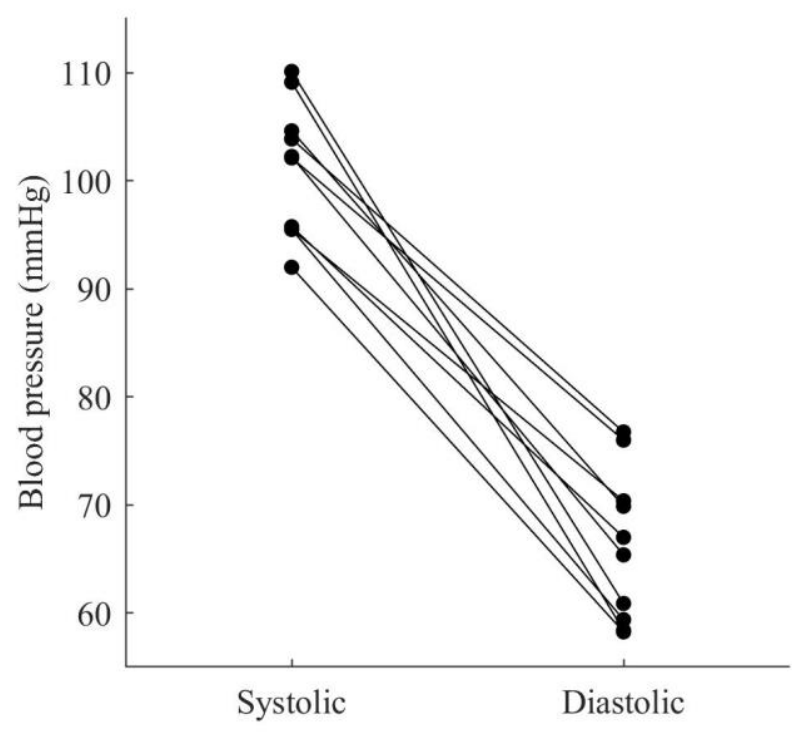

Figure 2. The average values for SBP and DBP for both operators and both measurements, plotted for all 10 subjects.
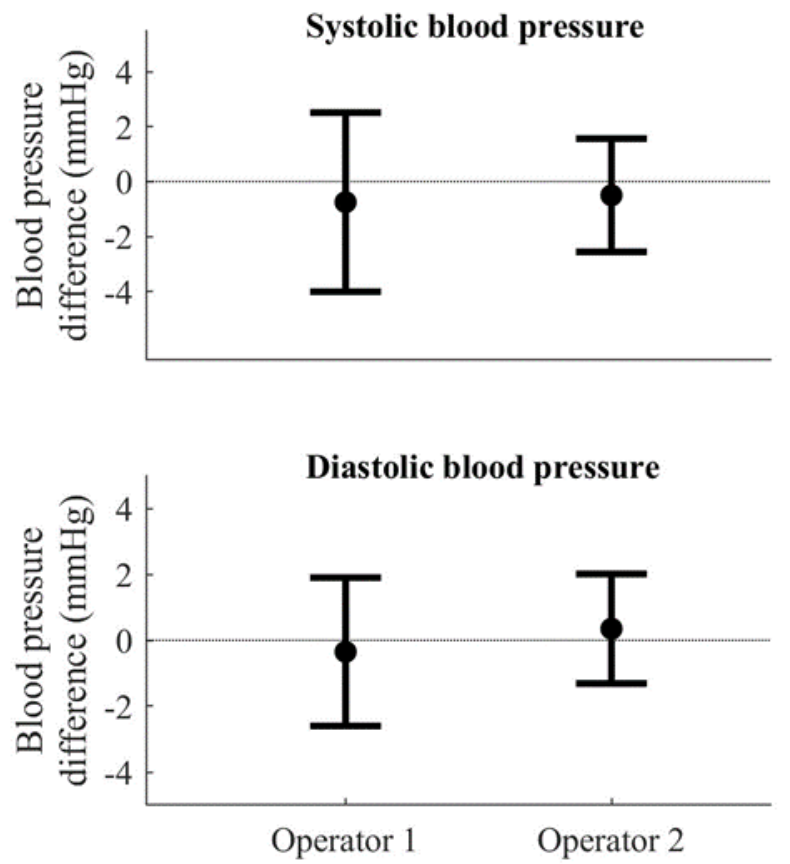

Figure 3. The blood pressure differences for repeat measurements from the same recordings, (mean \pm SD). Both measurements are included.
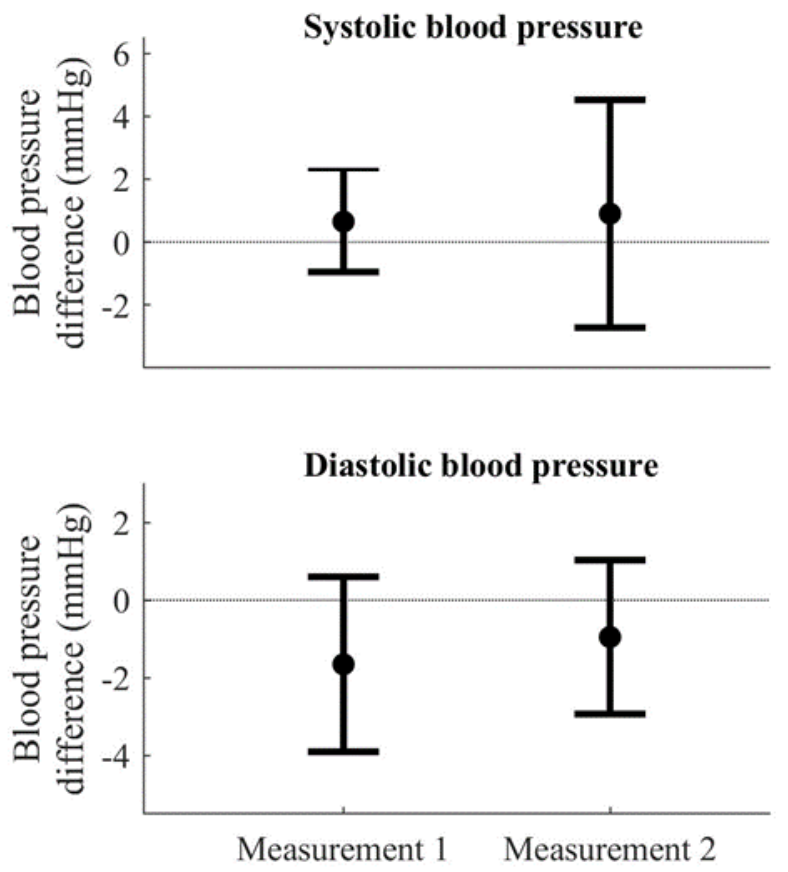

Figure 4. The blood pressure differences between the two operators, separately for the two measurement recordings, $($ mean $\pm \mathrm{SD})$. Both repeats are included.

\subsection{Difference between the two operators}

The difference between the two operators for each measurement was $0.7 \pm 1.6$ and $0.9 \pm 3.6 \mathrm{mmHg}$ for SBP, and $-1.7 \pm 2.3$ and $-1.0 \pm 2.0 \mathrm{mmHg}$ for DBP (all mean \pm $\mathrm{SD})$. This is shown in figure 4.

\section{Conclusion}

We have shown similar measurement variability between operators as between repeat measurements on identical recordings. We did, however, see a small difference between operators.

\section{Acknowledgment}

We acknowledge the UK Engineering and Physical Sciences Research Council (EP/1027270/1, EP/N025342/1) for their support for our blood pressure measurement research. 


\section{References}

[1] Laher M, O'Brien E. In search of Korotkoff. British Medical Journal 1982;285;1796-8.

[2] Grim CE, Grim CM. Auscultatory BP: still the gold standard. Journal of the American Society of Hypertension 2016;10:191-193.

[3] Graves JW, SG Sheps. Does evidence-based medicine suggest that physicians should not be measuring blood pressure in the hypertensive patient? Am J Hypertens 2004;17:354-360.

[4] Non-invasive sphygmomanometers - Part 2: Clinical investigation of intermittent automated measurement type. ISO 81060-2:2018

[5] Stergiou GS, Alpert B, Mieke S, Asmar R, Atkins N, Eckert $\mathrm{S}$, et al. A universal standard for the validation of blood pressure measuring devices: Association for the Advancement of Medical Instrumentation, European Society of Hypertension, International Organization for Standardization (AAMI/ESH/ISO) Collaboration Statement.

[6] https://bihsoc.org/resources/bp-

measurement/bpmeasurement-auscultatory-tutorials

Address for correspondence:

Prof Alan Murray

Engineering School and Medical Faculty

Newcastle University, UK

alan.murray@ncl.ac.uk 